\title{
Menstrual hygiene management in disasters: the concerns, needs, and preferences of women and girls in Vanuatu
}

\author{
Sandra G. Downing, Sandrine Benjimen, \\ Lisa Natoli, and Veronica Bell
}

\begin{abstract}
In disaster settings, women and girls face additional challenges in safely managing menstruation with confidence and dignity. Assessing women's and girls' needs and preferences at country level prior to a disaster assists Red Cross National Societies with preparedness planning and development of local menstrual hygiene management (MHM) kits for pre-positioning. This study, conducted in Vanuatu, examined the experiences, concerns, needs, and preferences of women and girls in managing menstruation in disasters. Participants trialled one of four different sanitary products included in a MHM kit. Absorbency, security of fit, and odour were the main determinants of product acceptability. Water supply and safe disposal options influenced the preference for either reusable or disposable products. Access to sanitary products, distribution processes, water supply, and privacy were key concerns. Detailed recommendations within this paper will support the Vanuatu Red Cross in decision making and programming for MHM in disaster preparedness and response.
\end{abstract}

Keywords: menstruation, menstrual hygiene management, MHM kits, disaster, Vanuatu, women and girls

MENSTRUAL HYGIENE MANAGEMENT (MHM) REFERS to a range of actions and interventions that ensure women and girls can privately and safely manage their menstruation. A comprehensive MHM approach involves not only access to MHM materials such as sanitary pads and underwear, but also access to appropriate infrastructure and menstrual health and hygiene education (Sommer et al., 2017).

MHM can be especially challenging for women and girls in low and middle-income countries, where discriminatory gender norms, societal taboos, and secrecy and embarrassment around menstruation are common (Hennegan et al., 2019). Disaster and displacement contexts exacerbate these challenges and include: lack of access to

Sandra G. Downing (sandra.downing@jcu.edu.au), Senior Lecturer, College of Public Health, Medical and Veterinary Sciences, James Cook University, Cairns, Australia; Sandrine Benjimen (centygal@gmail.com), Health Team Leader, Vanuatu Red Cross Society; Veronica Bell (vbell@redcross.org.au), Head of International Technical Services, Australian Red Cross Society; Lisa Natoli (Inatoli@redcross.org.au), Technical Health Lead, Australian Red Cross Society (c) The authors, 2021. This open access article is published by Practical Action Publishing and distributed under a Creative Commons Attribution Non-commercial No-derivatives CC BY-NC-ND licence http://creativecommons.org/licenses/by-nc-nd/4.0/. www.practicalactionpublishing.com 
sanitary materials, including underwear; lack of access to private/safe toilets and disposal options for used sanitary materials; lack of soap, water, private bathing facilities, and places to dry reusable sanitary materials; and reduced privacy and dignity, especially in overcrowded, temporary, and transit settings (Schmitt et al., 2017, VanLeeuwen and Torondel, 2018b).

Hygiene kits containing sanitary pads distributed during the acute phase of an emergency have attempted to partially address women's needs, but have many limitations. These kits are generally bulk procured outside the affected country, do not take into account the context-specific practices and preferences of women, and are distributed to families without consideration of the number of menstruating women within the family group (Giles-Hansen et al., 2019).

In recent years, greater recognition of the MHM challenges in disaster settings has informed guidelines and humanitarian standards (Sommer et al., 2017; Sphere, 2018). The International Federation of Red Cross and Red Crescent Societies (IFRC) have used this information to inform development of a MHM toolkit for Red Cross and Red Crescent Societies (IFRC, 2018). The toolkit advocates for the pre-positioning of MHM kits for distribution to individual women and girls in any emergency response. It also encourages assessment of women and girls' MHM preferences and practices at country level as part of preparedness planning, to guide country-specific MHM kit development.

Vanuatu, located on the earthquake prone 'ring of fire' and situated at the centre of the Pacific cyclone belt, consistently rates as the most disaster-prone country in the world (Day et al., 2019). Vanuatu Red Cross is highly experienced in responding to the needs of the most vulnerable in times of disasters and crises. Increasingly, the Vanuatu Red Cross team have been working to integrate MHM considerations into disaster preparedness and response programming.

In 2019, Vanuatu Red Cross collaborated with Australian Red Cross and James Cook University on a study to explore the socio-cultural aspects of menstruation likely to affect MHM in disaster settings and disaster-related MHM experiences, concerns, needs, and preferences of women and girls in Vanuatu. The objectives of the study were to identify preferred sanitary hygiene products and other menstrual hygiene management items for inclusion in local MHM kits, and to identify opportunities to strengthen Vanuatu Red Cross programming and interventions to support safe and dignified menstrual management in disaster settings. In this paper, we describe the findings of this study and the implications of this for disaster preparedness and response activities.

\section{Methods}

\section{Study setting}

The study took place at four sites, one urban and one rural on each of the islands of Efate and Espiritu Santo. Efate is home to an urban/progressive population in the capital Port Vila, and includes a number of communities impacted by Cyclone Pam in 2015. Espiritu Santo is typically more traditional and conservative than Efate. It is also home to a number of relocated/displaced communities. 


\section{Study design}

This was a concurrent mixed methods research study.

Focus group discussions (FGDs), including a participatory exercise to review the contents of the proposed MHM kits, and key informant interviews (KIIs) with a purposive sample of reproductive age women and girls were conducted. The kit was contained in a sturdy bucket with lid and included clothesline and pegs, underwear (two pieces), soap, laundry detergent, and a packet of one of four sanitary products selected to be trialled. The two disposable sanitary products (Maxfree and Modess) were chosen based on local availability and were considered most commonly used, acceptable, and affordable by the Vanuatu Red Cross project staff. Only one locally made reusable sanitary product is available in Vanuatu (Mamma's Laef), so a globally available, affordable product with established acceptability and efficacy (AFRIpads) was also chosen for trial.

A simple paper-based survey collected quantitative data as a supplement to the qualitative component of the study. The survey asked participants to indicate their level of satisfaction with the sanitary product they trialled using a Likert scale of emoji faces in relation to the following statements:

- They fitted into my underpants securely.

- They were comfortable and didn't irritate my skin.

- They were absorbent and didn't leak.

- They absorbed the blood smell.

- They were easy to change.

- They are a product I would recommend.

These statements were constructed from themes that emerged in previous studies trialling menstrual products (Mason et al., 2015; Kuncio, 2018).

\section{Participant recruitment}

Community engagement preceded participant recruitment in each of the project sites. Specific targeting of male Red Cross volunteers to support the community engagement strategy was undertaken. The engagement strategy was cognizant of the important role that men would play in community awareness and acceptance of the project, encouraging women's involvement in the study and supporting the idea of safe and dignified MHM for women. Vanuatu Red Cross staff and volunteer networks identified community groups such as women's or church groups and other groups that had previously engaged with Vanuatu Red Cross activities as potential sources for recruitment. An initial meeting introduced the research, providing the opportunity for discussion and questions. A community information sheet in the local language was also provided. Interested women and girls were invited to a further meeting to receive more detailed information on study requirements.

Eligible study participants were women and girls aged 15-45 years, who resided in the study sites, were willing to participate, and able to provide voluntary, informed consent. Potential participants were provided with a participant information and consent form in the local language and given time to review and ask questions. 
Where potential participants had low literacy, the form was read aloud by one of the researchers.

Purposive sampling facilitated the recruitment of girls and women into pre-specified participant groups in each location. Participants were grouped into three categories based on marital status and age (single, younger married, older married) in line with cultural norms. The aim of this grouping was to facilitate open discussion among peers during the FGDs and to ensure a broad range of girls and women were included in the study. Girls and women living with a disability that could affect MHM were actively sought to participate.

Following recruitment, participants were randomly allocated, within their grouping and location, to receive an MHM kit including one of the four sanitary products to trial over the timeframe of several menstrual cycles, across the months of June and July 2019. Each participant received verbal education on the use, care, and disposal of the allocated product by a member the research team. Written instructions by the manufacturers were also provided for the reusable products.

\section{Data collection/management}

Data collection occurred in August 2019. A small team comprising female Vanuatu Red Cross staff and volunteers, supported by a lead researcher from James Cook University (SD), collected the data. SD provided one day of training to the local team in Vanuatu, including the background and purpose of the research, principles of qualitative research, the role of different members in the research team (facilitators and note takers), and ethical considerations. The content of the FGD and interview guides were discussed and translated into the local language, Bislama. Participatory tools to support the FGDs were agreed upon and adapted to the Vanuatu context.

Data collection occurred in discreet and culturally appropriate locations in each community, facilitated by members of the local research team in Bislama. Telephone calls and/or text messages informed and reminded participants of the date, time, and location of the FGDs. FGDs and KIIs were digitally recorded with permission from participants. One local researcher was designated as facilitator for each FGD, one took notes in Bislama and another sat in the background with SD and translated as the discussion unfolded. This enabled SD to follow the discussion, determine interesting discussion points to probe or add to subsequent FGDs or interviews, and also take notes in English.

At the time of the FGDs and/or interview, participants were invited to complete an anonymous, short paper-based survey with questions specifically regarding their level of satisfaction with the sanitary product they trialled. Research team members assisted participants with lower levels of literacy to complete the survey.

\section{Ethical considerations}

The James Cook University (JCU) Human Research Ethics Committee and the ethics committee at the Vanuatu Ministry of Health reviewed and approved this study. 
All participants provided written informed consent. Local leadership/governance structures and culturally accepted concepts of adulthood guided the need to gain consent from family, parents or husbands. Where participants had low literacy or a physical disability that impaired their written capacity, the participant's verbal consent was witnessed by another adult and by a member of the research team and a declaration signed to this effect.

\section{Data analysis}

The research team undertook preliminary data analysis during fieldwork. This involved a brief meeting at the completion of each FGD and interview to compare notes, discuss emerging themes and refine question guides as appropriate.

At the completion of data collection, recordings of FGDs and interviews were transcribed verbatim into Bislama then translated into English. The lead researcher from Australia Red Cross (LN) read the transcripts in full to become familiar with the overall data set. Using an inductive approach, LN developed a coding framework that was independently verified by SD on a subset of transcripts, and the coding framework was then systematically applied to all transcripts. Coded sections of text were arranged according to overarching themes (Pope et al., 2000). Similarities and differences were explored within participant groups and across study sites.

Findings were summarized in a study report that was reviewed by the in-country research team for accuracy and feedback. The finalized report and recommendations were shared with stakeholders and communities in Vanuatu.

\section{Results}

Of the 192 women and girls recruited to the study and provided with an MHM kit, 136 took part in data collection. Twelve FGDs involving a total of 125 (65 per cent) participants were conducted. Discussions ranged in length from 38 to 107 minutes (average 77 minutes). Surveys were completed by 136 (71 per cent) participants.

Individual semi-structured interviews were conducted with two married, urban women living with disabilities. One woman had mobility issues and used a wheelchair and the other had impaired vision. Both women were invited to participate in FGDs but only one accepted.

\section{Participant characteristics}

Tables 1 and 2 provide the location and grouping of participants in the FGDs and survey respondents. Participants from the Efate urban site and single girls were least represented in the FGDs (Table 1). Single girls also had the lowest response rates to the survey (Table 2).

The two KIIs with women living with disability were conducted in the Efate urban site.

Of the 136 survey responses, 115 had a completed product rating section (Table 3). Eighteen participants did not have a menstrual cycle where they used the trial product and a further three participants did not complete the product rating section of the survey. 
Table 1 Focus group discussion participants by location and group

\begin{tabular}{lccccc}
\hline & $\begin{array}{c}\text { Older } \\
\text { married }\end{array}$ & $\begin{array}{c}\text { Younger } \\
\text { married }\end{array}$ & Single & Total & $\begin{array}{c}\text { Response } \\
\text { rate (\%) }\end{array}$ \\
\hline Efate urban & 7 & 9 & 7 & 23 & 48 \\
Efate rural & 16 & 11 & 7 & 34 & 71 \\
Santo urban & 11 & 13 & 8 & 32 & 67 \\
Santo rural & 11 & 15 & 10 & 36 & 75 \\
Total & 45 & 48 & 32 & 125 & 65 \\
Response rate (\%) & 70 & 75 & 50 & & \\
\hline
\end{tabular}

Table 2 Survey respondents by location and group

\begin{tabular}{lccccc}
\hline & $\begin{array}{c}\text { Older } \\
\text { married }\end{array}$ & $\begin{array}{c}\text { Younger } \\
\text { married }\end{array}$ & Single & Total & $\begin{array}{c}\text { Response } \\
\text { rate (\%) }\end{array}$ \\
\hline Efate urban & 14 & 9 & 15 & 38 & 79 \\
Efate rural & 13 & 13 & 5 & 31 & 65 \\
Santo urban & 14 & 9 & 9 & 32 & 67 \\
Santo rural & 7 & 16 & 10 & 33 & 69 \\
Not stated & 0 & 2 & 0 & 2 & - \\
Total & 48 & 49 & 39 & 136 & 71 \\
Response rate (\%) & 75 & 77 & 61 & & \\
\hline
\end{tabular}

Table 3 Survey responses by participant group and trialled sanitary product

\begin{tabular}{lcccc}
\hline & Older married & Younger married & Single & Total \\
\hline AFRlpad & 15 & 10 & 8 & 33 \\
Mamma's Laef & 9 & 7 & 13 & 29 \\
Modess/Softex & 9 & 6 & 9 & 24 \\
Maxfree & 7 & 15 & 7 & 29 \\
& 40 & 38 & 37 & 115 \\
\hline
\end{tabular}

\section{Findings}

Key themes and qualitative findings, together with key survey findings, are summarized below.

\section{MHM disaster-related experiences and concerns}

Access to absorbent materials. Access to sanitary pads was the most frequently mentioned concern, and a number of women gave examples of disaster experiences when sanitary pads were not provided by responding humanitarian organizations. Women noted that during rapid displacement they may forget to pack pads, and in the aftermath of a disaster, road and shop closures would affect 
availability. The expense of buying pads at a time when resources are scarce and need to be channelled into disaster recovery was also mentioned as an important consideration.

Access to water. Women explained that they use larger volumes of water when menstruating. This relates to preferences to shower more frequently and the need to wash stained underwear and clothes or reusable pads or absorbent materials.

It also relates to the common practice of rinsing out disposable pads prior to disposal, to discourage flies, and help ensure that male community members are not exposed to menstrual blood, which is considered shameful and offensive.

First I wash out the blood, then dry in the sun in a place where none of my brothers can see because of the respect we have for our brothers, then I burn it with rubbish. (Santo rural, older married)

Recognizing the increased demand for water during menstruation, but the common scarcity of water in displacement settings, many felt apprehensive about a negative response from men if women were seen to be using additional water. 'If we want to wash our panties and clothes soiled in blood, some men might say that we are wasting water and its disaster time we have to manage water' (Efate rural, young married).

Concerns for personal safety if there was a need to access water beyond the evacuation centre were also mentioned.

Safe disposal option. Participants worried about how they would dispose of used pads, especially if they had to share toilets with men.

Living in an evacuation centre, women and girls will have to find a safe, private place to safely burn [or] bury ... their pads. If sharing a toilet with men we cannot throw our pads in the toilet but we will have to walk into the bush to burn or bury [them] ourselves. (Efate urban, young single)

Privacy and dignity. The lack of privacy in evacuation centres was a major concern. This was mentioned both in relation to privacy for personal hygiene and while washing or disposing of pads.

Privacy will be a great challenge. Loss of dignity if I stain my clothes. Other people will see me as a dirty person and say that I am not capable of looking after myself. It will be a shameful thing for me. (Efate rural, young single)

There will be no privacy for women to change their pads and dispose safely. If I use calico [reusable pads], I will just save it and keep it in my bag until night time to wash and hang outside to dry. (Efate, Urban, young married)

Distribution processes. Participants spoke of their experiences receiving sanitary pads during relief operations, and indicated embarrassment about having MHM kits distributed along with other non-food items (NFIs).

I will be embarrassed if MHM kit is also distributed together [with] other NFIs [non-food items] because men and boys will be at the distribution point and they can make fun of us. (Efate urban, mixed) 
We will be happy [to receive MHM kits] but afraid of men asking us questions about menstruation and mocking us with our buckets. (Efate, Urban, older married)

Some also gave examples of receiving items during relief operations (such as reusable pads) that were unfamiliar to them, and being unsure about how to use and care for them.

\section{MHM needs and preferences in disaster settings}

MHM kits. Women who completed the paper-based survey indicated a good level of acceptability for all four sanitary products trialled (range: 80-97 per cent). The main determinants of product acceptability were absorbency and security of fit to underwear (to protect against leakage), and associated odour.

Of the two disposable options, a higher proportion of women who trialled Maxfree would recommend this product compared to the women who trialled Modess/ Softex (90 per cent versus 80 per cent). Both products had leakage issues with 29 per cent of women who trialled Modess/Softex and 34 per cent of women trialling Maxfree indicating they were neutral or unhappy with absorbency (Figures 1 and 2).

Of the two reusable options, a higher proportion of women who trialled AFRIpads would recommend this product compared with the women who trialled Mamma's Laef (97 per cent versus 86 per cent). Leakage was also noted as an issue with Mamma's Laef with 34 per cent of women who trialled this product indicating dissatisfaction with absorbency (Figures 3 and 4).

Additional feedback was gained from the FGDs and KIIs:

It is comfortable as well because it has its pin to clip it to the panty so it is stable and not moving. (Efate urban, young married)

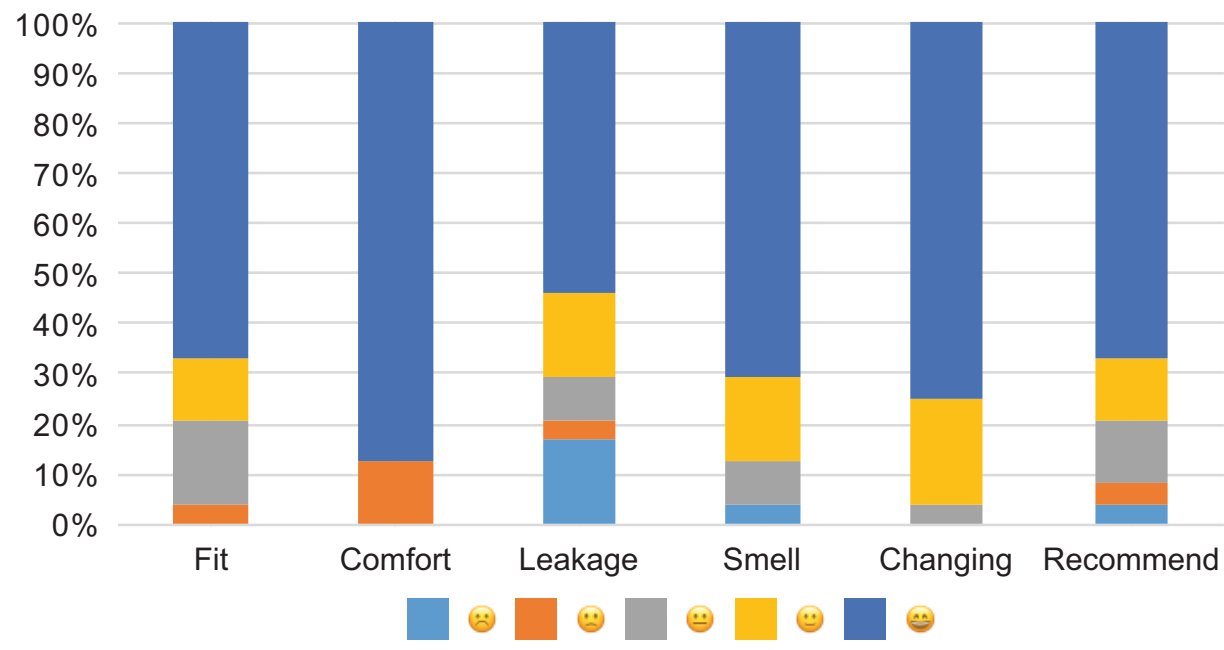

Figure 1 Modess product rating 
152 S. G. DOWNING ET AL.

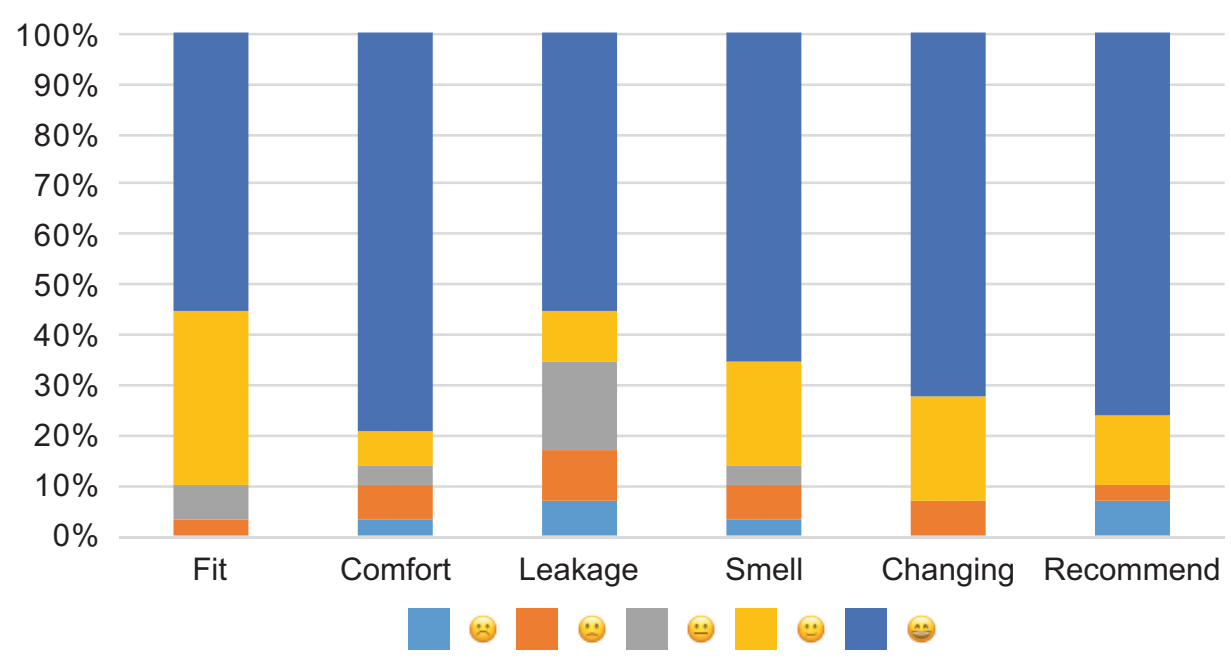

Figure 2 Maxfree product rating

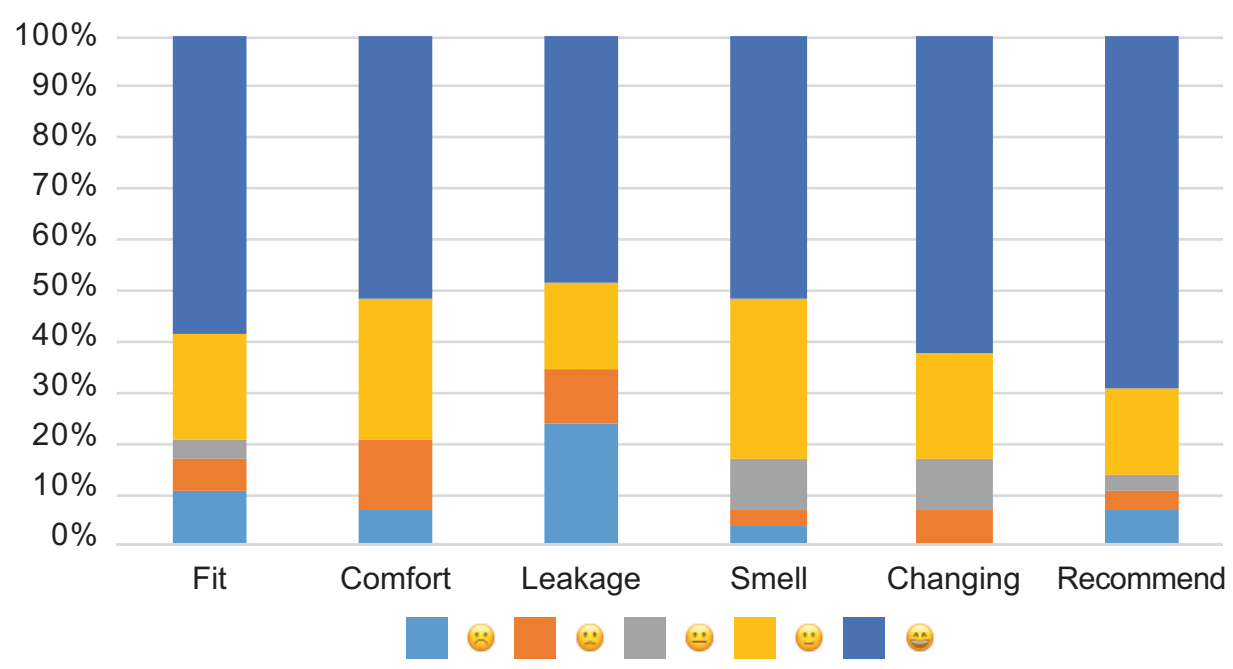

Figure 3 Mamma's Laef product rating

I can sleep comfortably till morning and I don't have to change at midnight. (Efate urban, young married)

I use Mamma's Laef but the only disadvantage is the smell during usage. So perfumes must be used to avoid shame. (Efate rural, young single)

Regardless of the type of pad provided (disposable or reusable), the need for an adequate supply was emphasized.

Many women were familiar with using hand sewn cloth pads, but few had experience using specifically designed reusable pads. The secure fit, absorbency, 


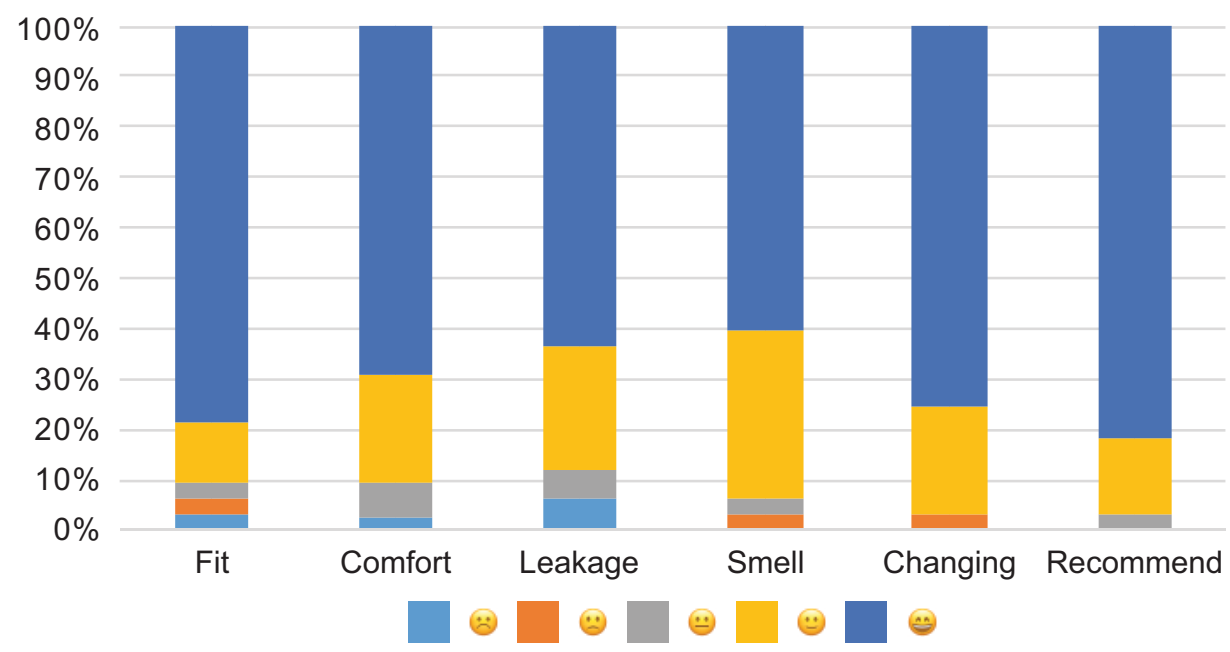

Figure 4 AFRIpad product rating

and ease of washing/drying were seen as very positive. Although both trial pads were generally regarded as quick drying there was concern that they would not dry as well during the wet season. There was a preference for colourful/patterned material that would hide stains. 'During sunny day it will dry fast but during rainy and cloudy weather it cannot dry easily' (Efate urban, mixed).

Most women were familiar with using disposable pads and while they were seen as an easier option in evacuation centres, some expressed concerns about their impact on the environment.

No need to wash and no need to find a private space to hang it so it is easy to manage your privacy at an evacuation centre because after use you just throw in the bush toilet. (Santo urban, Mango/Ngerngar mixed group)

It's not good for the environment when we throw it into the bush toilet or burn it. (Santo urban, mixed)

The context of the disaster and water availability came into consideration when determining the preferred sanitary product type. 'If there is water I prefer reusable and I can wash. If there's water shortage at the evacuation center I prefer disposable' (Efate urban, young single).

The two women living with disability expressed strong preferences for sanitary product type indicating a requirement for individual needs to be considered during distribution.

I heard the women say about the cloths to use but I hated it. I only like stayfree. (Woman, vision impaired)

We would talk about those things like how could you manage your monthly period and she [friend with hand disability] would say it just hard because her hands can't move properly. (Woman, wheelchair dependent) 
The additional materials included in the MHM kits were considered acceptable to participants; however they expressed preference in colour and size of 'panties' and also made suggestions for additional items to be included in the kit.

The nylon panties supplied were well received with numerous women noting they were soft, fast drying, and did not cause a rash. There was a strong preference for darker colours to hide stains. It was recommended that more than two pairs be included in the kit and that there be an option to choose the appropriate size. 'Black, red, brown, and dark colours is the best colours because stains are not visible on the panties' (Efate, urban, young married).

Suggestions for additional items included: a scrubbing brush; a dark coloured towel; a dark coloured lava lava (sarong); solar light and/or torch with spare battery; baby wipes (in event of water scarcity for personal hygiene); rubber gloves (for washing clothes); hand sanitizer (if water scarce); coconut oil or powder; and toilet paper. 'Tight fit pants' (similar to bicycle pants) to help keep pads in place were also suggested by the woman with mobility issues.

It was also suggested that information sheets provided with MHM kits should include information on the menstrual cycle and how to track days, personal hygiene measures and how to use, care for, and/or dispose of sanitary pads.

MHM kit distribution processes. Many women self-impose restrictions on their mobility during menstruation, largely due to fear of leakage/staining of clothes and the associated teasing and humiliation that can accompany this. 'When I have my period, I am usually shy to walk around in the community and participate in community activities because of the fear of staining myself in public' (Santo rural, older married).

This sensitivity may impact a woman's desire to take part in response and recovery activities such as distributions. Participants indicated a clear preference for MHM kit distribution to occur separately from the routine distribution of non-food items (NFI). It was suggested that ideally women would conduct the distribution; however, they said it would be acceptable for men to assist provided they were older and more mature so that there was no teasing. 'I feel more comfortable with women doing the distribution because I can easily ask MHM related questions to women instead of men' (Santo rural, older married).

It was felt that targeted awareness prior to distribution would motivate women and girls to attend a distribution to receive their individual MHM kits, but that special efforts would need to be made to reach younger girls with information.

Usually for us young girls we do not usually receive information, only women and mothers. So it is best to disseminate information through our youth committee so the information can reach every young girl within the community. (Efate urban, single)

It was also suggested that men be made aware of why women are receiving kits, so that they do not feel jealous or that they are missing out.

The challenges for women and girls with disabilities to attend distributions were noted, with household visits seen as the best option to address this. Community 
leaders and community groups were suggested as a useful entry point to identify these individuals.

It is best for the distribution team to visit each woman with disability at their household and provide awareness and also give them their MHM kit instead of having them and their caregiver coming to the distribution site. (Efate urban, young married)

Water, sanitation, and hygiene facilities. Participants noted the need for separate, safe facilities for women in evacuation centres and identified specific features to be considered. These included having a solid door with an inside lock, good lighting, adequate space for changing clothes if needed, rubbish bin inside, good ventilation, handwashing station with soap, and a private space for washing/drying pads and panties. 'The toilet must have a solid door with a lock on the inside and a light for use at night ... and a disposal container inside for rubbish' (Efate, rural, young single).

The specific needs of women and girls with disabilities were also considered. 'If there is a woman with disability, the toilet must have a ramp and a big space inside enough to fit the wheelchair' (Santo rural, older married).

In the absence of female friendly facilities, women suggested the need to support each other to ensure that MHM needs could be managed in privacy and safely.

During that time of disaster, we must help each other in time of menstruation. If someone sees her period, we must help take her to a place where it is safe ... away from where people live, to wash her clothes, swim and put on a new one [pad]. (Santo, rural, young married)

Involving women in disaster planning. The need for women to be involved in disaster preparedness and response planning so that their needs and concerns are taken into account was raised. At the same time, the value of sensitizing men to the MHM needs of women and girls was highlighted.

Normally in this village only men take part [in] disaster committee meeting but I think women should take part to share their concerns and needs and make their voice heard so agencies can better help address women and girls needs during a disaster. (Efate rural, young married)

... maybe humanitarian agencies can do a separate MHM awareness for men to help them understand why menstruation is a very important need for women. (Efate urban, mixed)

\section{Discussion}

Despite being an important issue impacting women and girls, menstrual hygiene has often been overlooked in disaster settings (Krishnan and Twigg, 2016). It is only in recent years that we have seen published studies of MHM experiences and linked interventions specific to humanitarian contexts (Budhathoki et al., 2018; Sommer et al., 2018; VanLeeuwen and Torondel, 2018a; Schmitt et al., 2021).

Access to absorbent materials is a significant concern for women and girls in disaster settings. Others have similarly found that sanitary products are often not 
included in relief packages, especially in the early weeks after a disaster (Budhathoki et al., 2018; Yoosefi Lebni et al., 2020), or that products are supplied per household, rather than according to the number of reproductive age females in that household (VanLeeuwen and Torondel, 2018b). The need for a sufficient supply of sanitary pads expressed by women in our study has been highlighted in previous studies (Schmitt et al., 2017; Kuncio, 2018; Kemigisha et al., 2020). Our study found drivers of sanitary product acceptability, notably absorbency and, in the case of reusable materials, the colour and ease of washing and drying, to be largely consistent with existing literature (Kuncio, 2018; VanLeeuwen and Torondel, 2018a).

Access to absorbent materials is not the only consideration, however, and humanitarian guidelines suggest that sanitary products should be provided with other supportive materials such as soap and underwear (Sommer et al., 2017). Importantly, it has been highlighted that culturally specific practices and preferences be understood in order to inform the range of supportive items that might be included in a local MHM kit (Sommer et al., 2017; VanLeeuwen and Torondel, 2018b). While our study identified suggestions and preferences specific to Vanuatu, such as a lava lava (sarong) to wrap around clothes to hide stains while walking to the bathroom, the range of items suggested for inclusion in a local MHM kit were broadly consistent with those included in guidelines (Sommer et al., 2017). Similarly, the identified needs and preferences related to female friendly WASH infrastructure, including safe disposal options, align with what has been described previously (Sommer et al., 2017; Kemigisha et al., 2020; Roxburgh et al., 2020).

Our study has strengthened understanding of women's and girl's preferences for how distribution processes should occur. It is noteworthy that distribution of MHM kits should be female led, be separate from distribution of other non- food items, and occur discreetly at a time and place that will not attract the attention of men and boys. At the same time, women recognized the need to engage men to help ensure an understanding of MHM needs, so, for example, they can appreciate the reasons behind female-focused distributions or be more sensitive to increased need for water while menstruating. The importance of male engagement to support MHM is well recognized (Giles-Hansen et al., 2019; Schmitt et al., 2021).

The Sendai Framework for Disaster Risk Reduction 2015-2030 calls for the empowerment and participation of women in disaster risk reduction, preparedness, and response (United Nations International Strategy for Disaster Reduction, 2015). However, case studies from disaster-prone regions have found that women's voices often remain unheard (Reyes and Lu, 2016; Dube and Mhembwe, 2019) and women have not felt able to express their MHM needs, or they have been overlooked by men in charge of distribution processes (Budhathoki et al., 2018; Yoosefi Lebni et al., 2020). The participants in our study, in one of the most disaster-prone countries in the world, have called for greater involvement of women and girls in disaster planning to ensure their needs and preferences are taken into account.

This study has a number of limitations. The chosen sample size, in terms of both number of sites and individual participants, reflects a pragmatic balance between the need for strong research and the financial realities and timeframe available for conducting field work of this nature. A degree of sampling bias can 
be expected when purposive recruitment is conducted by local staff using their organizational and personal relationships to reach community members. Study sites were selected across two islands and in both urban and rural settings to garner a diverse range of views; however the findings should be considered in context and not interpreted as being representative of the population as a whole. While it was planned for each focus group to only include women/girls from within their participant group (young single, young married, older married) there were some instances where focus groups contained a range of participants. This may have influenced the participants' desire to respond openly to questions, particularly for the younger girls. The facilitators were aware of this and made efforts to put all participants at ease. Focus group and interview recordings were transcribed into Bislama then translated into English by Vanuatu Red Cross staff and volunteers rather than professionals. The varying degree of experience with transcription and translation of the staff and volunteers may have resulted in loss of nuance in some instances. The research team discussions and note taking by the JCU researcher at the time of the fieldwork have assisted to counteract any loss of meaning in the translations.

Findings from this study have informed detailed recommendations that will support Vanuatu Red Cross in decision making and programming for MHM in disaster preparedness and response. A number of recommendations have been written into revised standard operating procedures (for example for emergency response assessments and distribution processes) to help ensure they are actioned. Findings have also been shared with key stakeholders in Vanuatu, including the WASH cluster, to help inform response operations.

Full implementation of the recommendations remains challenging. The procurement and pre-positioning of MHM kits as a preparedness measure is constrained by funding mechanisms. Positively, awareness activities and kit distribution were conducted by female Vanuatu Red Cross volunteers during the 2020 Tropical Cyclone Harold response while male volunteers provided logistical support and MHM awareness for male community members.

\section{Acknowledgements}

This study was a collaboration between the Australian Red Cross Society, the Vanuatu Red Cross Society and the College of Public Health Medical and Veterinary Sciences, James Cook University. Funding for this study was provided through Australia Red Cross's partnership with the Australian Department of Foreign Affairs and Trade (DFAT), with in-kind support from James Cook University.

We would also like to acknowledge and thank the Vanuatu Red Cross Society staff and volunteers who contributed to this study through community engagement, recruitment, data collection, and transcribing and translating. The research would not have been possible without the active involvement of participating communities and individuals. We are appreciative of their support and willingness to share their experiences and thoughts. Finally, we would like to acknowledge the support provided by the Vanuatu Ministry of Health. 


\section{References}

Budhathoki, S., Bhattachan, M., Castro-Sánchez, E., Sagtani, R., Rayamajhi, R., Rai, P. and Sharma, G. (2018) 'Menstrual hygiene management among women and adolescent girls in the aftermath of the earthquake in Nepal', BMC Women's Health 18: 33 <https://doi.org/10.1186/ s12905-018-0527-y>.

Day, S.J., Forster, T., Himmelsbach, J., Korte, L., Mucke, P., Radtke, K., Thielborger, P. and Weller, D. (2019) World Risk Report, Bündnis Entwicklung Hilft and Ruhr University Bochum Institute for International Law of Peace and Armed Conflict (IFHV), Berlin.

Dube, E. and Mhembwe, S. (2019) 'Heightening gender considerations for women in flood disaster response through resource allocation and distribution in Zimbabwe', International Journal of Disaster Risk Reduction 40: 101281 <https://doi.org/10.1016/j.ijdrr.2019.101281>.

Giles-Hansen, C., Mugambi, G. and Machado, A. (2019) 'Experiences from East Africa and lessons in addressing the menstrual hygiene needs of women and girls', Waterlines 38: 236-46 $<$ https://doi.org/10.3362/1756-3488.18-00023>.

Hennegan, J., Shannon, A.K., Rubli, J., Schwab, K.J. and Melendez-Torres, G.J. (2019) ‘Women's and girls' experiences of menstruation in low- and middle-income countries: a systematic review and qualitative metasynthesis', PLoS Medicine 16: e1002803 <https://doi.org/10.1371/ journal.pmed.1002803>.

IFRC (2018) Addressing Menstrual Hygiene Managment (MHM) Needs: Guide and Tools for Red Cross and Red Crescent Societies, Geneva: IFRC.

Kemigisha, E., Rai, M., Mlahagwa, W., Nyakato, V.N. and Ivanova, O. (2020) 'A qualitative study exploring menstruation experiences and practices among adolescent girls living in the Nakivale Refugee Settlement, Uganda', International Journal of Environmental Research and Public Health 17(18): 6613 <https://doi.org/10.3390/ijerph17186613>.

Krishnan, S. and Twigg, J. (2016) 'Menstrual hygiene: a "silent" need during disaster recovery', Waterlines 35: 265-76 <https://doi.org/10.3362/1756-3488.2016.020>.

Kuncio, T. (2018) Pilot Study Findings on the Provision of Hygiene Kits with Reusable Sanitary Pads: Testing the Appropriateness and Acceptability of AFRIpads Reusable Sanitary Pads in Southwestern (Ugandan) Refugee Context among Schoolgirls, Geneva: UNHCR.

Mason, L., Laserson, K.F., Oruko, K., Nyothach, E., Alexander, K.T., Odhiambo, F.O., Eleveld, A., Isiye, E., Ngere, I., Omoto, J., Mohammed, A., Vulule, J. and Phillips-Howard, P.A. (2015) 'Adolescent schoolgirls' experiences of menstrual cups and pads in rural western Kenya: a qualitative study', Waterlines 34: 15-30 <https://doi.org/10.3362/1756-3488.2015.003>.

Pope, C., Ziebland, S. and Mays, N. (2000) 'Qualitative research in health care: analysing qualitative data', BMJ 320: 114-6 <https://doi.org/10.1136/bmj.320.7227.114>.

Reyes, D.D. and Lu, J.L. (2016) 'Gender dimension in disaster situations: a case study of flood prone women in Malabon City, Metro Manila', International Journal of Disaster Risk Reduction 15: 162-8 <https://doi.org/10.1016/j.ijdrr.2015.11.001>.

Roxburgh, H., Hampshire, K., Kaliwo, T., Tilley, E.A., Oliver, D.M. and Quilliam, R.S. (2020) 'Power, danger, and secrecy: a socio-cultural examination of menstrual waste management in urban Malawi', PLoS One 15 <https://doi.org/10.1371/journal.pone.0235339>.

Schmitt, M.L., Clatworthy, D., Ratnayake, R., Klaesener-Metzner, N., Roesch, E., Wheeler, E. and Sommer, M. (2017) 'Understanding the menstrual hygiene management challenges facing displaced girls and women: findings from qualitative assessments in Myanmar and Lebanon', Conflict and Health 11: 19 <https://doi.org/10.7916/d8tb1kh2>. 
Schmitt, M.L., Wood, O.R., Clatworthy, D., Rashid, S.F. and Sommer, M. (2021) Innovative strategies for providing menstruation-supportive water, sanitation and hygiene (WASH) facilities: learning from refugee camps in Cox's bazar, Bangladesh', Conflict and Health 15: 10 <https://doi.org/10.1186/s13031-021-00346-9>.

Sommer, M., Schmitt, M.L. and Clatworthy, D. (2017) A Toolkit for Integrating Menstrual Hygiene Management (MHM) into Humanitarian Response, New York: Columbia University, Mailman School of Public Health and International Rescue Committee.

Sommer, M., Schmitt, M., Ogello, T., Mathenge, P., Mark, M., Clatworthy, D., Khandakji, S. and Ratnayake, R. (2018) 'Pilot testing and evaluation of a toolkit for menstrual hygiene management in emergencies in three refugee camps in Northwest Tanzania', Journal of International Humanitarian Action 3: 6 <https://doi.org/10.1186/s41018-018-0034-7>.

Sphere Association (2018) The Sphere Handbook: Humanitarian Charter and Minimum Standards in Humanitarian Response, 4th edition, Sphere Association, Geneva.

United Nations Office for Disaster Risk Reduction (2015) Sendai Framework for Disaster Risk Reduction 2015-2030.

VanLeeuwen, C. and Torondel, B. (2018a) 'Exploring menstrual practices and potential acceptability of reusable menstrual underwear among a Middle Eastern population living in a refugee setting', International Journal of Women's Health 10: 349-60 <https://doi.org/10.2147/IJWH. S152483>.

VanLeeuwen, C. and Torondel, B. (2018b) 'Improving menstrual hygiene management in emergency contexts: literature review of current perspectives', International Journal of Women's Health 10: 169-86 <https://doi.org/10.2147/ijwh.s135587>.

Yoosefi Lebni, J., Khorami, F., Ebadi Fard Azar, F., Khosravi, B., Safari, H. and Ziapour, A. (2020) 'Experiences of rural women with damages resulting from an earthquake in Iran: a qualitative study', BMC Public Health 20: 625 <https://doi.org/10.1186/s12889-020-08752-z>. 